\title{
PRRT2-dependent dyskinesia: cerebellar, paroxysmal and persistent
}

Cell Research (2018) 28:3-4. doi:10.1038/cr.2017.142; published online 17 November 2017

In an elegant publication in Cell Research, Tan and colleagues showed that ablation of PRRT2 in cerebellar granule cells is sufficient to induce paroxysmal kinesigenic dyskinesia. PRRT2 turns out to downregulate the presynaptic SNARE complex in granule cell axons, which in turn controls the activity patterns of Purkinje cells, the sole output of the cerebellar cortex.

It has recently been shown that patients with mutations in the proline-rich transmembrane protein 2 (PRRT2) can suffer from paroxysmal kinesigenic dyskinesia (PKD) [1], but the pathophysiological mechanisms underlying the relation between the genetic alterations and severe episodic attacks of dyskinesia and dystonia have remained unclear. To unravel these mechanisms, Tan and colleagues generated several mouse models harboring global neuronal or region-specific PRRT2 truncations [2]. Mutant mice with global or cerebellum-specific truncation, but not forebrain-specific truncation, exhibited, similar to the clinical features of PKD in patients, severe episodes of aberrant motor behavior including dyskinesia and dystonic postures following generalized seizures and hyperthermia. These manifestations, which point towards a prime role for cerebellar dysfunction in this form of PKD, were corroborated by electron microscopic and electrophysiological analyses of the cerebellar granule cell, i.e., parallel fiber, to Purkinje cell input (Figure 1). PRRT2 was found to be prominently present in the presynaptic segments of the parallel fiber terminals and stimulation of these fibers in mutants with truncated PRRT2 resulted in a transient biphasic elevation and suppression of simple spike firing of Purkinje cells, promoting abnormal firing patterns. Accordingly, truncation of PRRT2 increased the mEPSC frequency and number of docked vesicles at the parallel fiber to Purkinje cell input (Figure 1), which is in line with an inhibitory control function in neurotransmission at the presynaptic level. Indeed, using a diverse series of binding assays, the authors uncovered that PRRT2 inhibits several proteins of the presynaptic SNARE complex.

This paper provides the first direct evidence for a link between PRRT2associated dyskinesia and specific cerebellar presynaptic deficits. PRRT2 has been suggested before to play a role in the release machinery of presynaptic vesicles [3] and interact with the SNARE complex [4], but this interaction was never demonstrated to be involved in PKD, let alone to be critical for cerebellar function [5]. Interestingly, cerebellar deficits in Purkinje cell activity have recently also been shown to contribute to Rapid Onset Dystonian Parkinsonism (RODP), another paroxysmal movement disorder, which is caused by a mutation in a sodium-potassium ATPase pump [6]. By providing the next example of a paroxysmal movement disorder that primarily originates in the cerebellum, rather than in the basal ganglia-thalamic circuit, the current paper is further opening up the avenue of research that aims to elucidate how deficits in the activity of Purkinje cells and/or that of their inputs can lead to movement disorders like PKD and RODP. So far, various in vivo datasets indicate that bursty and irregular Purkinje cell activity can result in abnormal movements, including not only dystonic [7, 8] but also ataxic [9] movements. The current in vitro data obtained from PRRT2 mutants support this hypothesis in that the firing frequency of their Purkinje cell activity following optogenetic stimulation of their parallel fiber input was transiently and biphasically increased and decreased as compared to wild-type cells. Question remains, which factors determine the duration of these episodes (often tens of minutes rather than seconds)? Does it merely depend on the initial event that evoked the abnormal firing pattern of Purkinje cells, which could include for example a period with seizures, stress or hyperthermia [2], or does it also depend on internal amplification mechanisms, such as those that are mediated by the projections from the cerebellar nuclei neurons to the granule cells in the cerebellar cortex [10] (Figure 1)? Given the similarities in duration of the behavioral and cerebellar neuronal correlates of the paroxysmal attacks in RODP mice [7, $8]$ and that of the dysfunctional motor episodes in the murine models described in the paper by Tan and colleagues [2], it appears plausible that in both RODP and PKD prolonged interactive, yet erratic, firing patterns of Purkinje cells and their downstream targets in the nuclei underlie the pathological motor behavior.

Thus, future research on PRRT2- 


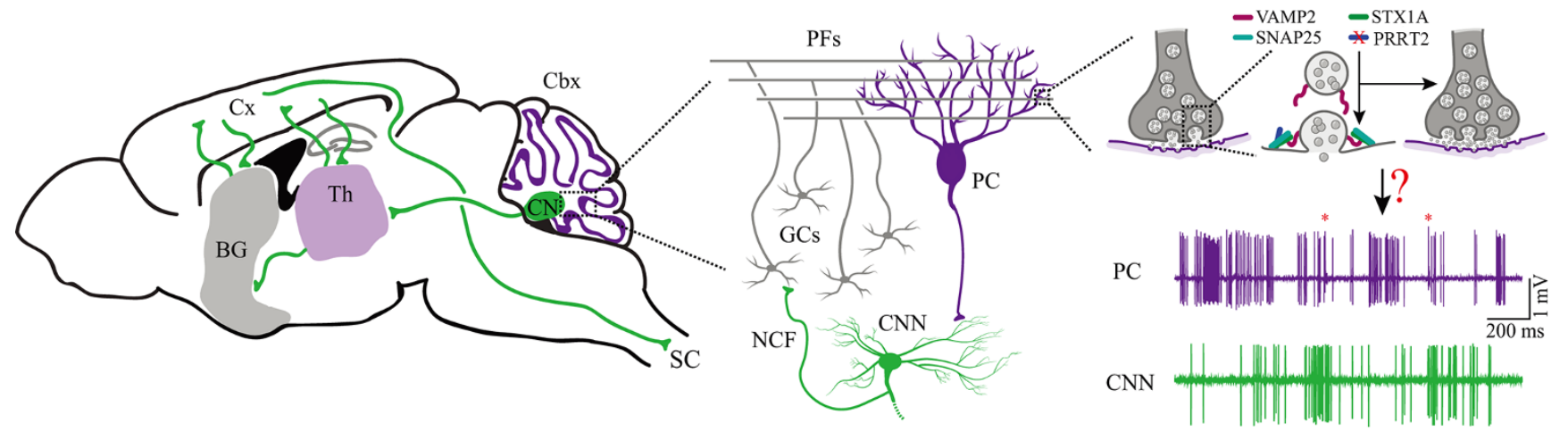

Figure 1 Potential cerebellar circuit mechanism contributing to PRRT2-dependent dyskinesia. The Purkinje cells (PCs) in the cerebellar cortex $(\mathrm{Cbx})$ inhibit neurons in the cerebellar nuclei $(\mathrm{CN})$, which in turn excite the thalamus (Th) and its networks with the cerebral cortex (Cx) and basal ganglia (BG) (left panel). The axons of the neurons in the cerebellar nuclei (CNN), which can drive motor behavior via these networks, provide recurrent nucleo-cortical fibers (NCF) innervating the granule cell layer in the cerebellar cortex (middle panel). This innervation may contribute to long-term changes in activity of the granule cells (GCs), which in turn modulate the PCs via their parallel fibers (PFs). As shown in the recent paper by Tan and colleagues, the transmission between PFs and PCs can be prominently affected by truncation of PRRT2. Ablation of PRRT2 results in reduced downregulation of the SNARE complex, an increase in docked vesicles and facilitated neurotransmission (right panel top). Upon stimulation, these changes in PF to PC transmission may lead to highly irregular cerebellar cortical and nuclear output (right panel bottom). Thus, once this activity in both the PCs and CNNs is aberrant, it may persist for long periods because of NCFs, generating a pathological reverberating loop prolonging the dyskinesia. Please note that the PC and CNN data in the right bottom panel were not recorded simultaneously and merely serve to exemplify the proposed irregularity; these data were obtained from tottering mice [11], which sometimes also exhibit similar paroxysmal dyskinesia attacks. The red asterisks above the example of irregular PC activity depict the occurrence of complex spikes. SC indicates the spinal cord, receiving input from Cx.

associated PKD should be aimed at bridging the gap in our current understanding at the in vitro and in vivo level, with specific emphasis on uncovering the circuit mechanisms that may explain the duration of the paroxysmal episode. First, combined interventional and electrophysiological experiments in vivo may unravel where the effects of distinct triggers like epileptogenic activity and hyperthermia converge with enhanced granule cell activity and lead to prolonged periods of putative irregular cerebellar firing. Next, simultaneous electrophysiological recordings of Purkinje cells and cerebellar nuclei neurons in awake behaving animals during the induction of dyskinesia attacks in vivo may reveal whether erratic interactions between the cerebellar cortex and nuclei indeed occur during episodes of PKD and whether the excitatory feedback is critical for its maintenance (Figure 1). By generating the current PRRT2deficient animal models exhibiting dyskinesia phenotypes and elucidating the primary molecular and cell physiological mechanisms, Tan and colleagues have provided an excellent framework to further expand upon with systemspathophysiological means.

\section{Lieke Kros $^{1}$, Chris I De Zeeuw ${ }^{1,2}$}

${ }^{1}$ Department of Neuroscience, Erasmus MC Rotterdam, the Netherlands; ${ }^{2}$ Netherlands Institute for Neuroscience, Royal Dutch Academy for Arts and Sciences, Amsterdam, the Netherlands Correspondence: Chris I De Zeeuw E-mail: c.dezeeuw@erasmusmc.nl

\section{References}

1 Chen WJ, Lin Y, Xiong ZQ, et al. Nat Genet 2011; 43:1252-1255.

2 Tan GH, Liu YY, Wang L, et al. Cell Res 2017; 28:90-110.

3 Valente P, Castroflorio E, Rossi P, et al. Cell Rep 2016; 15:117-131.

4 Stelzl U, Worm U, Lalowski M, et al. Cell 2005; 122:957-968.

5 Ebrahimi-Fakhari D, Saffari A, Westenberger A, et al. Brain 2015; 138:3476-3495.

6 Chen $\mathrm{CH}$, Fremont R, Arteaga-Bracho EE, et al. Nat Neurosci 2014; 17:1767-1775.

7 Fremont R, Calderon DP, Maleki S, et al. J Neurosci 2014; 34:11723-11732.

8 Isaksen TJ, Kros L, Vedovato N, et al. PLoS Genet 2017; 13:e1006763.

9 Hoebeek FE, Stahl JS, Van Alphen AM, et al. Neuron 2005; 45:953-965.

10 Gao Z, Proietti-Onori M, Lin Z, et al. Neuron 2016; 89:645-657.

11 Kros L, Eelkman Rooda OH, Spanke JK, et al. Ann Neurol 2015; 77:1027-1049. 